EPJ Web of Conferences 58, 02008 (2013)

DOI: $10.1051 /$ epjconf/20135802008

(C) Owned by the authors, published by EDP Sciences, 2013

\title{
Future sudden singularities in Palatini cosmology
}

\author{
Andrzej Borowiec ${ }^{2, a}$ and Michał Kamionka ${ }^{1, b}$ \\ ${ }^{1}$ Institute of Theoretical Physics, University of Wrocław \\ pl. Maksa Borna 9, 50-204 Wrocław, Poland. \\ ${ }^{2}$ Astronomical Institute, University of Wrocław \\ ul. Kopernika 11, 51-622 Wrocław, Poland.
}

\begin{abstract}
We show that future singularities which have appeared in the Palatini cosmological models investigated in [1] are of finite size at finite time type [2].
\end{abstract}

Doomsday scenarios for cosmological models are subject of numerous investigations and speculations. For example the standard LCDM model predicts a thermal death caused by the expansion forever. In this short note we solve similar problem for the Palatini cosmological models investigated already in [1].

First of all we recall that, similarly to LCDM case, Palatini models are described by dynamical systems of Newtonian type. They are fully determined by effective potential functions $V(a)$ defined by Friedmann equation (see [1]). The evolution of the Universe described by such system is given by:

$$
\frac{\dot{a}^{2}}{2}=-V(a) \Rightarrow \ddot{a}=-\frac{\partial V}{\partial a}
$$

where $a$ denotes the cosmic (FRWL) scale factor. From the potential plot (fig . 1) one can see that:
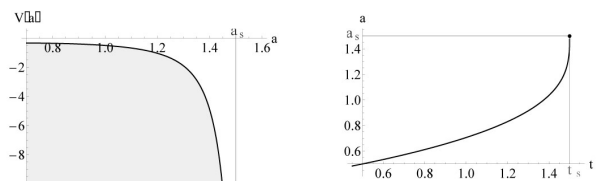

Figure 1. Left panel: typical future singularity of the potential function for cosmological models presented in [1]. Right panel: example of time dependency of the scale factor near singularity.

$$
\lim _{a \rightarrow a_{s}^{-}} V(a) \rightarrow=-\infty, \quad \lim _{a \rightarrow a_{s}^{-}} \ddot{a}>=+\infty
$$

as well as

$$
\lim _{a \rightarrow a_{s}^{-}} \dot{a}=+\infty
$$

for some finite $a_{s}<\infty$ singular (final) point.

\footnotetext{
ae-mail: borow@ift.uni.wroc.pl

be-mail: kamionka@astro.uni.wroc.pl
}

This is an Open Access article distributed under the terms of the Creative Commons Attribution License 2.0, which permits unrestricted use, distribution, and reproduction in any medium, provided the original work is properly cited. 
The question is whether the singular (finite) point has to be reached at finite time $t_{s}<\infty$. Assuming (a'contrario) $\lim _{t \rightarrow+\infty} a(t)=a_{s}^{-}$gives rise to

$$
\lim _{t \rightarrow+\infty} \dot{a}(t)=0^{+}
$$

which contradicts with (3). Therefore $t_{s}$ has to be finite (sudden).

As illustrative examples of time (analytic) dependency nearby singular point one can consider the family of functions (see (fig. 1) and [2])

$$
a(t)=a_{s}-b\left(t_{s}-t\right)^{\alpha}
$$

with $b>0$ and $0<\alpha<1$. Thus $\dot{a}=b \alpha\left(t_{s}-t\right)^{\alpha-1}$ and $\ddot{a}=b \alpha(1-\alpha)\left(t_{s}-t\right)^{\alpha-2}$. It is worth to mention that typical values of $a_{s}$ obtained in [1], after constraining model parameters by astrophysical data, are $1.4<a_{s}<2.5$.

\section{Acknowledgments}

M.K. is supported by the Polish NCN grant PRELUDIUM 2012/05/N/ST9/03857.

\section{References}

[1] A. Borowiec, M. Kamionka, A. Kurek and M. Szydlowski, JCAP 1202, 027 (2012) [arXiv:1109.3420 [gr-qc]].

[2] J. D. Barrow, Class. Quant. Grav. 21, L79-L82 (2004). [gr-qc/0403084];

S. Nojiri, S. D. Odintsov, S. Tsujikawa, Phys. Rev. D71, 063004 (2005). [hep-th/0501025];

M. P. Dabrowski and T. Denkiewicz, AIP Conf. Proc. 1241 (2010) 561 [arXiv:0910.0023 [gr-qc]];

J. D. Barrow, S. Cotsakis and A. Tsokaros, [arXiv:1301.6523 [gr-qc]]. 